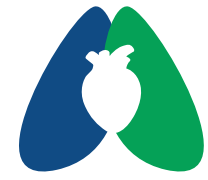

ASSOBRAFIR C I Ê N C I A

\section{Impacto de dois protocolos de estimulação diafragmática elétrica transcutânea nos parâmetros ventilométricos de pacientes críticos}

\author{
Impact of two protocols of transcutaneous electrical diaphragmatic \\ stimulation in the ventilatory parameters of critical patients
}

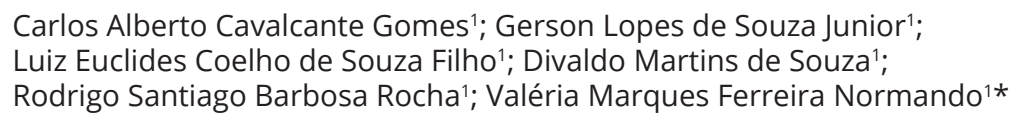

\begin{abstract}
Resumo
Introdução: a Ventilação Mecânica é um dos principais fatores que desencadeiam a atrofia muscular diafragmática na Unidade de Terapia Intensiva, correlacionada ao aumento do tempo de internação hospitalar, morbidade e mortalidade. A Fisioterapia pode minimizar essas complicações, otimizando a função muscular diafragmática, utilizando a estimulação diafragmática elétrica transcutânea (EDET). Objetivo: verificar o efeito de dois protocolos de EDET em pacientes em ventilação mecânica sobre parâmetros ventilométricos. Método: ensaio clínico randomizado, longitudinal, prospectivo, quantitativo, realizado em uma Unidade de Terapia Intensiva. Quinze participantes de ambos os sexos foram distribuídos aleatoriamente em: Grupo Experimental I (GE-I; $n=5 ; 64,80 \pm 5,07$ anos); Grupo Experimental II (GE-II; n=4; 68,50 \pm 7,51 anos) e Grupo Controle (GC; $n=6 ; 65,33 \pm 7,15$ anos). O protocolo terapêutico os condicionou às sessões de fisioterapia de rotina do hospital, porém para os GE-I e II foi acrescido protocolos da EDET em cinco sessões. A avaliação dos parâmetros ventilométricos foi direcionada pela ventilometria para acompanhamento do volume minuto $\left(V^{\prime}\right)$, volume corrente (VC) e frequência respiratória (FR) na avaliação inicial (T0) e final (T5) de cada atendimento no turno vespertino. Resultados: para a análise intergrupos apenas o VC apresentou significância estatística $(p<0,01)$, sendo maior no GE-II. Na análise intragrupo utilizando-se como referência os T0 e T5, houve resposta significativa para o VC no $G C(p=0,04)$, com piora após os dias de protocolo, e V' no GE-II $(p<0,01)$, com aumento significativo após as 10 sessões. Conclusão: a EDET foi capaz de aumentar parâmetros ventilométricos em pacientes em ventilação mecânica, o que pode indicar manutenção da função muscular após a realização do protocolo.
\end{abstract}

Palavras-chave: Respiração Artificial; Terapia por Estimulação Elétrica; Diafragma; Fisioterapia.

\begin{abstract}
Introduction: mechanical ventilation is one of the main factors that triggers diaphragmatic muscle atrophy in the Intensive Care Unit, correlated with the increase in the hospitalization period, morbidity and mortality. Physiotherapy can minimize these complications by optimizing diaphragmatic muscle function using Transcutaneous Electrical Diaphragmatic Stimulation (TEDS). Objective: to verify the effect of two TEDS protocols on ventilometric parameters in patients on mechanical ventilation. Methods: randomized, longitudinal, prospective, quantitative clinical trial, carried out in an Intensive Care Unit. Fifteen participants of both sexes were randomly assigned to: Experimental Group I (EG-I; n = 5; $64.80 \pm 5.07$ years); Experimental Group II (EG-II; $n=4 ; 68.50 \pm 7.51$ years) and Control Group (CG; $n=6$; $65.33 \pm 7.15$ years). The therapeutic protocol conditioned the groups to routine physiotherapy sessions at the hospital, however, for EG-I and II, TEDS protocols were added in 10 sessions. The evaluation of ventilometric parameters was guided by ventilometry to monitor the minute volume, tidal volume $\left(\mathrm{V}_{\mathrm{T}}\right)$ and respiratory rate $(\mathrm{RR})$ in the initial (T0) and final (T5) assessment of each appointment in the afternoon shift. Results: for the intergroup analysis, only the $\mathrm{V}_{\mathrm{T}}$ showed statistical significance $(\mathrm{p}<0.01)$, being higher in the $\mathrm{EG}-\mathrm{II}$. In the intragroup analysis using T0 and T5 as a reference, there was a significant response to $V_{T}$ in the CG ( $p$ $=0.04)$, worsening after the protocol days; and minute volume in the EG-II $(p<0.01)$, with a significant increase after 10 sessions. Conclusion: TEDS was able to increase ventilometric parameters in patients on mechanical ventilation, so it can indicate maintenance of muscle function after performing the protocol.
\end{abstract}

Keywords: Artificial Respiration; Electrical Stimulation Therapy; Diaphragm; Physiotherapy. sob a mesma licença 


\section{INTRODUÇÃO}

A Ventilação Mecânica (VM) tem como finalidade o suporte aos pacientes com incapacidade de manter adequada ventilação alveolar. Estudos prévios têm identificado uma significativa taxa de prevalência na necessidade do suporte ventilatório, onde aproximadamente $90 \%$ dos pacientes críticos necessitam de VM e o processo da retirada do suporte ventilatório representa $40 \%$ do tempo total do seu uso ${ }^{1-3}$.

A VM é um dos principais fatores na promoção da atrofia, fraqueza e alterações funcionais das fibras diafragmáticas. Desta forma, a prevenção da atrofia por desuso muscular e fraqueza deve ser iniciada precocemente, impactando no tempo de VM e internações na UTI. Para minimizar a ocorrência destes acometimentos, a realização de exercícios e técnicas de condicionamento e fortalecimento muscular respiratório podem ser relevantes ${ }^{4}$.

Neste sentido, a fisioterapia deve buscar o mais precocemente, estimular os pacientes submetidos à VM, a fim de minimizar a perda da massa e força muscular. Dentre seus recursos, destaca-se a técnica denominada de Estimulação Diafragmática Elétrica Transcutânea (EDET), que por meio de eletrodos colocados em pontos motores, gera potenciais de ação no nervo frênico a fim de estimular as fibras diafragmáticas à contração, efetivando a função do músculo diafragma ${ }^{5,6}$.

A EDET permite o recrutamento de maior número de fibras musculares íntegras do músculo diafragma, promovendo a contração muscular específica e consequente fortalecimento muscular, em especial nos pacientes com disfunções neuromusculares ${ }^{7}$.

Os efeitos da EDET possuem relação com as variáveis respiratórias, entretanto, existe uma diferenciação entre os equipamentos e os parâmetros da corrente elétrica, e até mesmo dos protocolos de intervenção, variando de acordo com os autores e o tipo de análise utilizada ${ }^{8}$.

Dentre as alternativas para avaliar as variáveis respiratórias durante a utilização da EDET encontra-se a ventilometria, que pode verificar importantes valores relacionados ao esforço voluntário do paciente, como volume corrente, volume minuto e frequência respiratória, sendo estes diretamente relacionados com a capacidade do diafragma e demais músculos respiratórios em expandir os pulmões sob uma demanda necessária por um determinado período de tempo9.

Sendo assim, uma maior abordagem científica sobre a EDET em pacientes críticos nas UTI's se apresenta como ponto de interesse a este estudo, que tem como objetivo verificar o efeito de dois protocolos da EDET sobre os parâmetros ventilométricos de pacientes submetidos à ventilação mecânica.

\section{MÉTODOS}

\section{Desenho do estudo}

Ensaio clínico randomizado, longitudinal, prospectivo, quantitativo e de centro único e registrado (NCT03712215) e com aprovação do Comitê de Ética e Pesquisa com Seres Humanos (parecer $n^{\circ} 1.762 .021$ ).

\section{Participantes e randomização}

Quinze participantes em suporte ventilatório mecânico invasivo fizeram parte do estudo, após assinatura, por parte do responsável, do Termo de Consentimento Livre e Esclarecido (TCLE). Os participantes passaram pelo esquema de randomização, planejado por um profissional que não participou das sessões de tratamento, com o programa "randomization.com", distribuindo os indivíduos aleatoriamente nos grupos do estudo, por meio de uma sequência numérica aleatória em envelopes lacrados e opacos. O terapeuta responsável pelo tratamento, antes de iniciar as intervenções, abriu o envelope contendo o grupo correspondente a cada participante. Os envelopes lacrados ainda não utilizados ficaram guardados em um local seguro e concedidos ao terapeuta responsável à medida que as avaliações foram ocorrendo.

A randomização dos participantes consistiu na formação de três grupos: Grupo Experimental I (GE I; $n=5$ ) onde foram submetidos ao protocolo de EDET baseado nos estudos de Geddes et al. ${ }^{10}$; Grupo Experimental II (GE II; $n=4)$, onde foram submetidos ao protocolo de EDET baseado nos estudos de Cancelliero et al.8; Grupo Controle (GC; $n=6)$ onde não foram submetidos a EDET. Todos os participantes dos grupos receberam atendimento fisioterapêutico protocolar, por parte da equipe de fisioterapia, de acordo com as condutas do manual de normas e rotinas do serviço de Fisioterapia da UTI.

Foram incluídos no estudo voluntários de ambos os sexos, com idade entre 60 e 80 anos, em ventilação mecânica invasiva por mais de 48 horas, com drive respiratório, fazendo uso de VM na modalidade ventilação em pressão suporte (VPS), conectado ao tubo orotraqueal ou por meio da cânula de traqueostomia, hemodinamicamente estáveis, ausência de sedativos e que o responsável legal ou voluntário tenha assinado o TCLE.

Foram excluídos do estudo voluntários que utilizavam marca-passo, com hipertermia (temperatura $>38^{\circ} \mathrm{C}$ ), com infecção sistêmica, que estivessem utilizando bloqueadores neuromusculares, com drenos torácicos, edema generalizado e com lesão cutânea na área de intervenção terapêutica com EDET.

Os participantes passaram por um protocolo de avaliação fisioterapêutica inicial na clínica/setor de UTI adulto da FHCGV, para os registros de identificação, anamnese e exame físico. Ressaltando que a cada sessão os mesmos eram reavaliados. As intervenções ocorreram 
em cinco sessões consecutivas, uma vez ao dia, no turno vespertino.

\section{Intervenção}

Para o procedimento de EDET foi utilizado o modelo Orion TENS II (Orion-SP-Brasil). O equipamento permite ser modulado livremente e foi aplicado no GE-I sendo utilizado no modo FES sincronizado, de acordo com os parâmetros baseados nos estudos de Geddes et al. ${ }^{10}$ : frequência da corrente (F) em $30 \mathrm{~Hz}$; largura de pulso (T) de 0,4 ms; tempo de subida (Rise) de 1s; tempo da contração muscular (On time) de $1 \mathrm{~s}$; tempo de descida (Decay) de $2 \mathrm{~s}$ e tempo de relaxamento muscular (Off time) de $1 \mathrm{~s}$.

No GE-Il foi utilizado o mesmo equipamento, diferenciando-se nos parâmetros que são baseados de acordo com os estudos de Cancelliero et al. ${ }^{8}$ para o procedimento de EDET, sendo utilizado no modo FES sincronizado, com frequência de $30 \mathrm{~Hz}$; largura de pulso (T) de 0,4 ms, subida (rampa) de 0,7 s (valor máximo). A sustentação foi de 0,4 s, já padronizada e fixa no aparelho.

Nos dois protocolos, a intensidade da corrente utilizada foi mínima para obter a contração do músculo diafragma, causando sensação confortável ao participante e com tempo de estimulação de 20 minutos por sessão. Sendo assim, a diferença foi nos tempos de subida e sustentação. Além disso, o tempo de descida não está presente no protocolo de Cancelliero et al. ${ }^{8}$, ou seja, após a sustentação há o repouso.

Para a colocação dos eletrodos, a pele foi previamente higienizada com álcool $70 \%$, onde com uma fita tipo micropore $(3 \mathrm{M} \AA)$ quatro eletrodos, de silicone-carbono de $5 \times 5 \mathrm{~cm}$, foram fixados com gel condutor incolor entre as superfícies, paralelos ao sentido das fibras do músculo diafragma. Sendo assim, foram posicionados dois eletrodos em cada lado do tórax, um par na região paraxifóidea direita ( $3^{\circ}$ espaço intercostal (EIC)) e linha axilar média direita $\left(7^{\circ} \mathrm{EIC}\right.$ ) e outro par na região paraxifóidea esquerda $\left(3^{\circ} \mathrm{EIC}\right)$ e linha axilar média esquerda ( $\left.7^{\circ} \mathrm{EIC}\right)$, locais dos pontos motores do músculo diafragma, padronizado nos estudos de Cancelliero et al. ${ }^{8}$ e Geddes et al. ${ }^{10}$.

Cada aplicação teve duração de 20 minutos, com os participantes posicionados em decúbito dorsal (cama elevada à $45^{\circ}$ ), cabeça centralizada, sem utilização de travesseiro e com os membros superiores e inferiores estendidos ao longo do corpo.

\section{Procedimento de coleta de dados}

Para a avaliação do volume minuto $\left(V^{\prime}\right)$ foi utilizado o ventilometro analógico Wright (Spire-SP-Brasil). Paralelamente foi observada a frequência respiratória (FR); em seguida volume corrente (VC), considerando-se V' = FR $x$ VC. Esta análise ocorreu durante a avaliação inicial e ao final do protocolo. Para a mensuração, o ventilômetro foi conectado em um filtro da marca HMEF UNDIS MEDICAL $®$ para adultos com traqueia extensível de $15 \mathrm{~cm}$, e esta acoplada diretamente na cânula de traqueostomia ou tubo orotraqueal, onde o participante permaneceu por um tempo de no máximo um minuto fora da VM.

A mensuração foi realizada três vezes, com intervalo de repouso de dois minutos entre cada, sendo considerado para o estudo o registro mais significativo. Foi considerado $25 \mathrm{ml}$ por incursão respiratória referente ao espaço morto do circuito filtro-traqueia.

As medidas ventilométricas foram verificadas pelo mesmo pesquisador em todas as etapas de coleta. Durante a realização do procedimento, no caso de instabilidade hemodinâmica ou queda de saturação periférica de oxigênio $\left(\mathrm{SpO}_{2}\right)$ para valores menores ou iguais a $90 \%$, o protocolo era imediatamente interrompido, retornando após condições clínicas estáveis. Após o final de cada atendimento os participantes retornavam aos cuidados da equipe do local, assim como ao fim do protocolo. Ressalta-se que todas as medidas de biossegurança foram cuidadosamente garantidas.

\section{Análise dos dados}

O tratamento estatístico dos dados foi realizado por meio do pacote estatístico SPSS 22.0, onde se adotou a estatística descritiva para a caracterização da amostra, através da média e o desvio padrão, e a estatística inferencial foi realizada através do teste de ANOVA one way para comparar as médias das diferenças pré e pós-tratamento entre os três procedimentos adotados, além do teste $t$ de Student para variáveis pareadas, para comparar as médias pré e pós-tratamento dentro de cada procedimento adotado. Foi adotado um nível de significância de $p \leq 0,05$ para as inferências estatísticas.

\section{RESULTADOS}

A amostra final da investigação foi constituída por 15 participantes, sendo oito do sexo masculino (53,3\%) e sete do feminino $(46,7 \%)$, distribuídos em GC ( $n=6)$ com idade de 65,33 \pm 7,15 anos; GE I ( $n=5)$ com idade de 64,80 $\pm 5,07$ anos e GE II (n=4) com idade de 68,50 \pm 7,51 anos (Tabela 1).

Na Figura 1 se observa um fluxograma referente aos voluntários da pesquisa, 23 participantes passaram pelo processo de avaliação para elegibilidade, porém oito acabaram por ser excluídos.

A Tabela 2 mostra as médias e desvios padrão das diferenças entre o pré e pós protocolo de intervenções diárias nos três grupos investigados e a comparação entre os grupos.

A análise intragrupo para o GC demonstrou diferença significativa $(p<0,01)$ somente para a variável VC, demonstrando piora dos valores entre o período pré protocolo $(443,63 \pm 83,20 \mathrm{ml})$ e o período pós protocolo (364,31 $\pm 74,86$ ml-). A análise das variáveis no GE-I não demonstrou significância estatística quando feita a comparação intragrupo. Quanto ao grupo GE-Il somente 


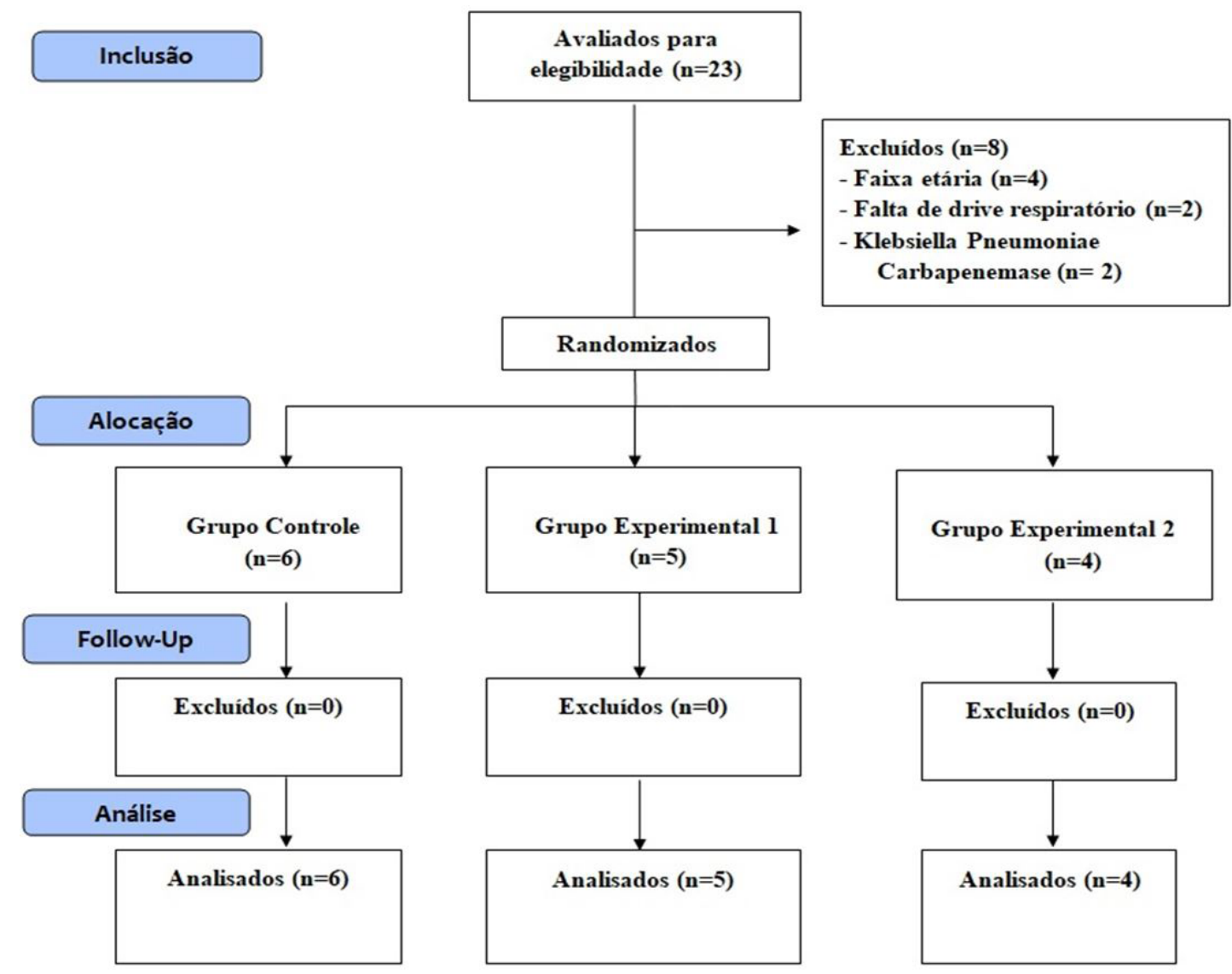

Figura 1. Fluxograma dos participantes da pesquisa.

Tabela 1. Prevalência das idades, gêneros e patologias dos grupos estudados.

\begin{tabular}{|c|c|c|c|}
\hline \multirow{2}{*}{ Grupos } & GC & GE-I & GE-II \\
\hline & $(n=6)$ & $(n=5)$ & $(n=4)$ \\
\hline Idade "anos" & $65,33 \pm 7,15$ & $64,80 \pm 5,14$ & $68,50 \pm 7,51$ \\
\hline Gênero (M/F) & $03 / 3$ & $03 / 2$ & $02 / 2$ \\
\hline \multirow{4}{*}{$\begin{array}{l}\text { Diagnóstico } \\
\text { clínico }\end{array}$} & Choque Séptico: 3 & Infarto Agudo do Miocárdio: 3 & Infarto Agudo do Miocárdio: 2 \\
\hline & Hérnia Inguinal Escrotal: 1 & Sepse: 1 & Sepse: $\mathbf{2}$ \\
\hline & Crise Convulsiva: 1 & DPOC exacerbado: 1 & \\
\hline & Infarto Agudo do Miocárdio: 1 & & \\
\hline
\end{tabular}

GC: grupo controle; GE-I: grupo experimental I; GE-II: grupo experimental II; M: masculino: F: feminino.

Tabela 2. Análise da ventilometria antes e após protocolos de intervenção.

\begin{tabular}{|c|c|c|c|c|c|c|}
\hline & $V^{\prime}(m L)$ & P intra & VC $(m L)$ & P intra & FR (irpm) & $P$ intra \\
\hline GC pré & $7755,00 \pm 1803,86$ & 0,75 & $443,63 \pm 83,20$ & $<0,01$ & $17,67 \pm 3,88$ & 0,14 \\
\hline GC pós & $7998,33 \pm 1654,30$ & & $364,31 \pm 74,86$ & & $22,33 \pm 5,96$ & \\
\hline GE-I pré & $9473,00 \pm 2752,92$ & 0,07 & $430,88 \pm 85,15$ & 0,14 & $21,80 \pm 3,11$ & 0,55 \\
\hline GE-I pós & $10428,00 \pm 2617,12$ & & $456,56 \pm 108,97$ & & $22,80 \pm 1,10$ & \\
\hline GE-II pré & $9858,33 \pm 831,16$ & 0,04 & $638,57 \pm 73,27$ & 0,89 & $15,00 \pm 2,83$ & 0,40 \\
\hline GE-II pós & $12225,00 \pm 556,78$ & & $647,26 \pm 2,02$ & & $18,50 \pm 0,71$ & \\
\hline P inter & 0,28 & & $<0,01$ & & 0,29 & \\
\hline
\end{tabular}

GC pré: grupo controle período pré protocolo; GC pós: grupo controle período pós protocolo; GE-I pré: grupo experimental I período pré protocolo; GE-II pré: grupo experimental II período pré protocolo; GE-II pós: grupo experimental II período pós protocolo; V': volume minuto; VC: volume corrente; FR: frequência respiratória; $P$ intra: valor de significância intragrupo; $P$ inter: valor de significância entre os grupos. $P<0,05$. 
a variável V' demonstrou valores significativos $(0,04)$ entre o período pré protocolo $(9858,33 \pm 831,16 \mathrm{ml})$ e o período pós protocolo $(12225,00 \pm 556,78 \mathrm{ml})$.

Aanálise entre os grupos para as variáveis ventilométricas estudadas demonstrou valores significativos somente para o volume corrente $(p<0,01)$, demonstrando maiores valores nos grupos GE-I $(456,56 \pm 108,97 \mathrm{ml})$ e GE-II $(647,26$ $\pm 2,02)$, quando comparados ao GC $(364,31 \pm 74,86 \mathrm{ml})$ no período pós protocolo.

\section{DISCUSSÃO}

O presente estudo propôs verificar o efeito de diferentes protocolos de EDET sobre variáveis ventilométricas de pacientes em ventilação mecânica. Pode-se identificar que os protocolos que utilizaram a EDET se sobrepuseram ao protocolo que não utilizou a estimulação elétrica transcutânea como estratégia de manutenção do trofismo muscular diafragmático.

Os resultados obtidos após a intervenção acerca de V', VC e FR, quando comparado entre os grupos, corroboram com os achados na literatura, a exemplo dos estudos de Santos et al. ${ }^{11}$, os quais utilizaram a EDET para melhorar a função respiratória em 21 idosos saudáveis, do sexo masculino, com faixa etária compreendida entre 67 e 79 anos, não fumantes, e sem história de doença pulmonar, neuromuscular ou alterações posturais. Ao final do estudo a EDET promoveu incremento no VC.

Esses achados também confirmam os estudos de Forti ${ }^{12}$ o qual analisou os efeitos da EDET na função pulmonar e na mobilidade toracoabdominal de pacientes obesas mórbidas submetidas a cirurgia bariátrica, e observou que a EDET se mostrou eficaz na manutenção dos fluxos e volumes pulmonares no pós-operatório.

Desse modo, evidenciaram-se ganhos significativos de VC e o V' nos pacientes que utilizaram tal recurso, corroborando com os achados de Forti et al. ${ }^{13}$, onde foram avaliados 20 indivíduos jovens e saudáveis, que utilizaram o EDET com resposta significativa dos VC e V'.

Com os resultados obtidos foi possível ratificar as pesquisas de Cancelliero et al. ${ }^{8}$ os quais puderam inferir que a EDET foi uma possível alternativa terapêutica para recuperar a condição clínica de limitações na mobilidade toracoabdominal e fraqueza muscular inspiratória tendo em vista que a mesma está diretamente relacionada ao VC e o V', observada em pacientes com DPOC.

Ainda de acordo com Cancelliero et al. ${ }^{8}$ a EDET tende a produzir uma contração por meio de uma corrente elétrica, com resposta sincrônica em todas as unidades motoras do músculo, provocando o recrutamento de fibras musculares diafragmáticas antes inativas. Devido a isso, este recurso fisioterapêutico é uma especificidade utilizada para melhorar a função ventilatória, auxiliando pacientes com fraqueza dos músculos respiratórios ou submetidos à $\mathrm{VM}^{14}$.
Segundo Baltieri et al. ${ }^{15}$, que foi apontado por um relato de caso, em uma paciente do sexo feminino, de 37 anos, admitida para revascularização do miocárdio, após intervenção com EDET, notou-se aumento gradual do VC, capacidade vital (CV), capacidade inspiratória (Cl) e força muscular respiratória, e a diminuição gradual do V' e da FR, e a manutenção do pico de fluxo expiratório (PFE).

Nos estudos de Ferreira et al. ${ }^{16}$, onde foi realizado um treinamento muscular respiratório utilizando a EDET, verificou-se um incremento importante do VC, o que segundo a pesquisa está relacionado a contrações mais fortes e efetivas da musculatura diafragmática facilitando assim a ventilação pulmonar e ampliando o volume de ar que transita por estas vias.

O GE-II apresentou resultados mais promissores em relação aos demais grupos, fato que pode ter ocorrido pois neste grupo a EDET não utilizou tempo de descida, sendo utilizado somente no GI-E. A modulação da rampa permite graduar a duração do pulso elétrico em um determinado tempo, fato que pode permitir aumento ou diminuição da contração muscular, sendo assim este grupo pode ter-se beneficiado mais em relação aos outros por permitir este controle e melhor estimulação das unidades motoras ${ }^{17}$.

Embora a EDET seja um recurso pouco estudado e com poucos dados na literatura científica, tem sido utilizada na prática clínica da fisioterapia, porém sem um protocolo com evidências científicas. Tais aspectos justificaram a realização deste estudo, devido à contribuição para fundamentação de protocolos na prática clínica.

Cabe salientar as limitações da presente pesquisa, no que se refere ao tamanho amostral, participantes com condições clínicas diversas, com predomínio de intervenções cirúrgicas e de doenças respiratórias, sendo que a maioria dos participantes apresentavam mais de uma patologia associada, o que pode ter influenciado nos achados quando comparados os grupos.

Além deste fato, existem muitos empecilhos que dificultam a realização de pesquisas com EDET em UTI, como a pluralidade de patologias encontradas neste local, a evolução rápida de pacientes que acabam por ser retirados da VM antes da finalização do estudo além de outros fatores referentes ao cotidiano da UTI que podem interferir nos resultados. Sendo assim, é importante que sejam realizadas mais pesquisas com um número maior de indivíduos para verificar seus efeitos mediante diferentes patologias, para que assim haja um consenso referente aos parâmetros mais eficazes em sua utilização.

\section{CONCLUSÃO}

Pode-se observar que os protocolos que utilizaram eletroestimulação levaram ao aumento de variáveis ventilométricas, sendo que os grupos GE-I e GE-II aumentou o volume corrente em relação ao GC, além disso o GE-II levou a melhora do volume minuto em relação aos valores iniciais. Seu potencial ainda não foi totalmente 
explorado, haja vista que não há uma grande quantidade de estudos acerca deste assunto encontrados nas bases de dados pesquisadas.

\section{FONTE DE FINANCIAMENTO}

Bolsa de Iniciação científica com apoio do Conselho Nacional de Desenvolvimento Científico e Tecnológico CNPq.

\section{CONFLITO DE INTERESSES}

Os autores declaram não possuir conflito de interesse

\section{REFERÊNCIAS}

1. Barbas CSV, Ísola AM, Farias AMC, Cavalcanti AB, Gama AMC, Duarte ACM, et al. Recomendações brasileiras de ventilação mecânica 2013. Parte I. Rev Bras Ter Intensiva. 2014;26(2):89-121. http://dx.doi.org/10.5935/0103507X.20140017. PMid:25028944.

2. Shimizu JM, Manzano RM, Quitério RJ, Alegria VTC, Junqueira TT, El-Fakhouri S, et al. Fatores determinantes da mortalidade em ventilação mecânica e os efeitos de dois protocolos de desmame respiratório. MTP\&Rehabjournal. 2014;12:377-94.

3. Meireles FMS, Barbosa IO, Viana MCC, Kuehner CP. Caracterização de parâmetros e estratégias do desmame difícil da ventilação mecânica adotados por fisioterapeutas. Rev Bras Promoç Saúde. 2013;26(1):51-5. http://dx.doi. org/10.5020/18061230.2013.p51.

4. Mendez-Tellez PA, Needham DM. Early physical rehabilitation in the ICU and ventilator liberation. Respir Care. 2012;57(10):1663-9. http://dx.doi.org/10.4187/ respcare.01931. PMid:23013903.

5. Dall'Acqua AN. Estimulação elétrica neuromuscular preserva morfologia da musculatura abdominal e peitoral de pacientes críticos em ventilação mecânica [dissertação]. Porto Alegre: Universidade Federal do Rio Grande do Sul; 2015.

6. Queiroz AGC, Silva DD, Lira RAC, Bassini SRF, Uematsu ESC. Treino muscular respiratório associado à eletroestimulação diafragmática em hemiparéticos. Rev Neurociênc. 2014;22(2):294-9. http://dx.doi.org/10.34024/rnc.2014. v22.8101.

7. Martinelli B, Santos IP, Barrile SR, Iwamoto HCT, Gimenes C, Rosa DMC. Estimulação elétrica transcutânea diafragmática pela corrente russa em portadores de DPOC. Fisioter Pesqui. 2016;23(4):345-51. http://dx.doi.org/10.1590/18092950/14854823042016.

8. Cancelliero KM, Ike D, Sampaio LMM, Santos VLA, Stirbulov R, Costa D. Estimulação diafragmática elétrica transcutânea (EDET) para fortalecimento muscular respiratório: estudo clínico controlado e randomizado. Fisioter Pesqui. 2012;19(4):303-8. http://dx.doi.org/10.1590/S180929502012000400002.

9. Vasconcelos TB, Costa RB, Josino JB, Magalhães CBA, Maia NPS, Bastos VPD. Análise da função pulmonar em indivíduos com lesão do plexo braquial. Rev Neurociênc. 2015;23(4):48692. http://dx.doi.org/10.4181/RNC.2015.23.04.1110.07p.

10. Geddes LA, Voorhees WD, Bourland JD, Riscili CE. Optimum stimulus frequency for contracting the inspiratory muscle with chest-surface electrodes to produce artificial respiration. Ann Biomed Eng. 1990;18(1):103-8. http:// dx.doi.org/10.1007/BF02368420. PMid:2306029.

11. Santos LA, Borgi JR, Daister JLN, Pazzianotto-Forti EM. Efeitos da estimulação diafragmática elétrica transcutânea na função pulmonar em idosos. Rev Bras Geriatr Gerontol. 2013;16(3):495-502. http://dx.doi.org/10.1590/S180998232013000300008.

12. Forti EMP. Estimulação diafragmática elétrica transcutânea (EDET) em mulheres obesas mórbidas submetidas à derivação gástrica em $Y$ de Roux (DGYR) com anel de contenção, por laparotomia [tese]. São Carlos: Universidade Federal de São Carlos; 2008.

13. Forti EMP, Pachani GP, Montebelo MIL, Costa D. Eletroestimulação diafragmática transcutânea em indivíduos saudáveis. Rev Fisioter Bras. 2018;6(4):261-4. http://dx.doi. org/10.33233/fb.v6i4.2003.

14. Santos LA, Silva CA, Polacow ML. Efeito do tratamento precoce com estimulação diafragmática elétrica transcutânea (EDET) na inflamação pulmonar provocada pela Bleomicina. Braz J Phys Ther. 2013;17(6):606-13. http://dx.doi.org/10.1590/ S1413-35552012005000130. PMid:24346295.

15. Baltieri L, Santos LA, Pessotti E, Forti EMP. Estimulação diafragmática elétrica transcutânea na paralisia diafragmática após cirurgia cardíaca. Rev Bras Cardiol. 2012;25(6):504-6.

16. Ferreira L, Mello J, Brito M, Cavenaghi O. Efetividade da estimulação diafragmática elétrica transcutânea na força muscular respiratória, volumes e capacidades pulmonares: revisão sistemática. Medicina. 2015;48(5):491-500.

17. Borges F, Souza F, Oliveira J, Evangelista A. Parâmetros de modulação na eletroestimulação neuromuscular utilizando corrente russa. Rev Fisioter Ser. 2007;2(1):1-10. 